\title{
“Because You Can Make Things with It": A Rationale for a Project to Teach Mathematics as a Multimodal Design Tool in Secondary Education
}

\author{
Susan Gerofsky \\ University of British Columbia
}

\begin{abstract}
This paper reports on the rationale for a new collaborative project at the University of British Columbia to develop multisensory software for secondary school mathematics learning. The project is described with reference to related mathematical, haptic, kinesthetic and musical software development for mathematics learning and in light of justifications for an embodied, multisensory, and fluidly translatable mathematics immediately applicable by students in purposeful ways.
\end{abstract}

This paper offers a rationale and description of a newly-launched multifaceted collaborative design project for secondary school mathematics education. I will begin by outlining two big-picture problems in secondary mathematics noted by many educators: first, that many students have great difficulties dealing with an increasingly-abstract math curriculum as they progress through high school mathematics classes, and second, that traditionally-taught secondary school math classes do not address the modes of learning and being that secondary students live in their lives outside of math class, and thus miss many opportunities for engaging students in the learning of mathematics. An anecdote from the author's own teaching experience is offered as a source of suggested approaches to the two problems introduced above.

The rationale for a multisensory, fluidly multimodal approach to teaching secondary mathematics is outlined in terms of a review of theoretical and applied work already in progress in this area and some hypothetical questions the collaborative team is using to guide our design process. At the end of this paper, a description of the scope of this project and some details of its (proposed or realized) implementation are offered as an example of an integrated approach to the issues raised.

\section{Problems Addressed by the Project}

\section{Problem 1: Learners Find Wholly Abstract Learning Difficult}

Mathematics is involved in the business of perceiving, abstracting and extending patterns. By secondary school, much of the mathematics that students encounter is encoded in terse and elegant algebraic formulations that express these patterns in the most economic symbolism possible. For many students, questions of learning style and cognitive

Susan Gerofsky is an Assistant Professor of mathematics education at the University of British Columbia in Vancouver, British Columbia. 
developmental maturity make the highly abstract forms of traditional mathematics instruction hard to grasp.

Large numbers of secondary students encounter tremendous difficulty working in solely abstract, symbolic terms (Tall \& Thomas, 1991). This difficulty is not simply correlated with students' inherent mathematical ability or willingness to learn mathematics; for both more and less successful secondary mathematics students, the emphasis on mathematical abstraction, beginning with the introduction of algebra, brings problems with building robust concepts. When the sole point of access to new concepts is an algebraic formula, many students stop trying to form a deep understanding of new ideas, and instead resort to rote learning and procedural routines ("plugging in formulas") as a survival technique.

\section{Problem 2: Traditional Secondary School Mathematics Teaching Misses Opportunities by Ignoring New Ways of Learning and Being}

Students growing up in a networked world of converging computerized technologies are not served well by a mathematics curriculum served up in lectures and premade algorithms, to be noted down, memorized and applied correctly on tests. The efficient industrial centralized delivery model of mathematics education, if it ever worked, does not work now in the decentralized, complex knowledge networks of our internet-based society. In realms outside of school mathematics classes, learners act as authors, teachers, publishers, makers, collaborators, scavengers, bricoleurs, publishers-of videos, music, graphics, text, websites, animations, games, photos, magazines, clothing, objects - and most often, several of these in combination. This playful, artful making and sharing of ideas, objects, texts, images and sounds is almost invariably mediated by electronic technology focused on computer networks, but it is also characterized by a fluid movement among virtual and physically present worlds and among various software and hardware tools.

The predominant modes of being and learning in our contemporary world are characterized by fluid and multiple identities (actual and virtual), learning and teaching as an in-depth, obsessive experience enmeshed in the formation of temporary or long-lasting social networks and friendships, a cavalier attitude to actual physical embodiment, which has become obsolete, optional, and thus an art form (McLuhan \& McLuhan, 1988), and constant multitasking, experienced either as a successful juggling of a multiplicity of tasks or a stress-inducing lack of single focus and constant distraction (Menzies 2005).

Embodiment has become a central issue to our contemporary culture. As Marshall McLuhan predicted (for example, McLuhan \& McLuhan, 1988) and postmodern theorists have further elaborated (for example, Haraway, 1985), the possibility of living in a disembodied, multibodied, cyborg or engineered mode has made the "natural" body obsolete, optional, an art form. We are all experiencing this world where identities are variable, fluid and liable to theft; where bodies are a canvas for artwork (tattoos, piercings, cosmetic surgery) or are neglected (a prevalence of obesity); where face-to-face meetings are optional, and become a kind of cool, retro art form. In this culture, the notion of body becomes a central issue, and an easy, playful movement between virtual and physical bodies is the new "natural." 
Much of secondary school mathematics education has placed itself outside this world, the world that learners and teachers inhabit. Traditionalists claim either to represent timeless perennial truths and essential knowledge using timetested means (and those students who cannot learn by these means are obviously not fit to learn mathematics at all); or they claim to provide a haven of rational thought based on the application of pencil-and-paper algorithms and proofs, away from the hurly burly of the irrational world - a refuge of clear right and wrong answers, infallible authority and logical certainty.

Although most visits to secondary school math classrooms in North American schools reveal an ongoing fidelity to these Platonist, perennialist, essentialist traditions established in public schooling over a century ago, there are important currents pulling math education in other directions. Most influential and notable is the work of the National Council of Teachers of Mathematics (NCTM), whose new standards and principles for math education are based on a constructivist, or perhaps a social constructionist philosophy of mathematics learning. The NCTM cites technology use in mathematics teaching and learning as a foundational principle, and mathematical communication, problem solving and connections as process standards for education (NCTM 2000). There is a concerted effort by the NCTM (and educators committed to its program) to overcome the inertia of long-established teacher-centred practice and bring in a reform of teaching, learning and assessment in math education.

Our project is not the only one developing multisensory computer applications for mathematics education. Several research teams in computing science and mathematics in a variety of locations have independently begun to work on haptic (tangible) and auditory (mostly musical) computer-human interfaces for the purpose of teaching particular topics in mathematics and physics (Williams, Chen \& Seaton, 2000; Treviranus, 1999). Most of these teams are concentrating on multisensory math learning for visually impaired students (Van Scoy, McLaughlin \& Fullmer, 2005; Van Scoy, Kawai, Darrah \& Rash, 2001; Ramloll et al., 2000; Roth, Petrucci \& Pun, 2000). The TERC institute in Boston lists a completed project, "Using Haptic Devices to Learn Mathematics and Science", with project director Ricardo Nemirovsky (who headed a recent Research Forum at PME on Perceptuo-Motor Activity and Imagination in Mathematics Learning (Nemirovsky, 2003)). As haptic technology becomes cheaper, more available and more compact, interest in developing educational applications for this new interface will likely grow.

\section{Theoretical and Experiential Grounding}

This project takes the following ideas as central organizing premises in addressing the problems initially raised:

- Mathematics learning for deeper, relational understanding (Skemp, 1976) necessarily involves shuttling back and forth between concrete and abstract representations of mathematical patterns, and between different kinds of representations of these patterns (for example, graphs, models, equations, tables of values, aesthetically-pleasing graphic and concrete embodiments of these relationships, dynamic digital models, etc.) "Deep understanding” in mathematics can be at 
least partially understood as the ability to conceive of a wide variety of representations or phenomena as structurally equivalent instances of a particular mathematical pattern, which can also be expressed and manipulated abstractly (Thurston, 1995). The greater the number and variety of representations available, the easier it is to build robust concepts through a variety of entry points and equivalences for a particular pattern or concept.

- Subordination of specific instrumental math skills to an overarching task that uses those skills (for example, solving an interesting larger problem, or using the mathematics as a design tool) allows for "practice through progress" (Hewitt, 1996). The subordinated skills will be learned in more lasting and thorough way when they are not the focus of conscious attention than through repeated meaningless practice drills.

- Embodied mathematics is a stage in learning more abstract mathematical concepts, and may be revisited recursively at many points in the process of learning and problem-solving. With a recognition that mathematics is not a product of pure, disembodied reason but is rather grounded in our human, sensory and physical experiences of the world, there has been a call for the recognition of embodied concepts as an important initial stage in the formation of mathematical concepts (Tall, 2004; Tall. 2003; Radford, 2003; Pimm \& Jackiw, 2002; Tall, 2002; Watson \& Tall, 2002; Lakoff \& Nuñez, 2000). Particular developments in the history of mathematics have been analyzed as embodied concepts (Lappas \& Spyrou, 2003; Nuñez, 2000; Nuñez, Edwards \& Matos, 1999).

- Research mathematicians use embodied and visual models as an essential part of building their own research understandings. Research mathematicians and epistemologists of mathematical scholarship have noted that, even when the final results are published in the terse and economical format of abstract equations, mathematical researchers use visualization and embodied mathematical concepts (including drawings, physical models and computer models) as essential tools for reasoning, exploring and communicating with colleagues about developing mathematical ideas (Henderson \& Taimina, 2007; Whiteley, 2002, 2004; Burton, 2001).

- Through embodiments of mathematical objects students can have access to the aesthetics of mathematics. Recent work has developed the aesthetic as a facet of school mathematics (Sinclair, Pimm \& Higginson, 2007; Sinclair, 2005; Gerofsky, Sinclair \& Davis, 2002; Schroeder \& Gerofsky, 1998). A sense of beauty, elegance, aptness and sensory pleasure motivate learning, and are aspects of mathematics often pointedly ignored in secondary mathematics. (For evidence of this, one need only visit the typically grey, bare boxes of rooms in which mathematics is taught in secondary schools.) Aesthetic/ multisensory perceptions can help learners perceive patterns that might not be available from, say, a list of numbers (Sinclair, 2005; Van Scoy, McLaughlin, \& Fullmer, 2005).

This cluster of foundational ideas for the project was brought home for me in the following experience from my own practice as a high school teacher some years ago, when I took on the job of teaching a Grade 9 (age 14) information 
technology class halfway through the year. My experiences with this class convinced me of the potential for robust and engaged mathematics learning when the following elements are brought into play in a school setting:

- $\quad$ students using mathematics as a means for their own ends

- mathematics learning subordinated to a design purpose

- $\quad$ engagement with virtual as well as physically-present worlds

- multiple representations of mathematical objects or concepts

- mathematics closely linked with the aesthetics of representation

- $\quad$ sensory and (imaginatively) embodied connections with mathematical representations

The students in my IT class had learned Pascal programming and were designing their own video games. Many of the games involved projectiles moving under the force of gravity. For example, one student was making a game where the player controlled a stick figure throwing a basketball through a hoop. The students had learned how to program an object moving along a straight line trajectory, but this was obviously unsatisfactory for the parabolic path of a basketball arcing up and falling. Other students encountered similar problems with bouncing, shot or thrown objects in their games.

According to the provincial math curriculum, the topic of quadratic functions was not to be taught to these kids for another year. Nonetheless, there was a clear, felt need for these students to learn about parabolas and their transformations. Where it is sometimes difficult to motivate students to learn about quadratic equations in math class, this group of students was very eager to know how to use quadratics, with a purpose in mind - to improve their games. They demanded to learn how to change equations to move parabolas around the screen, make them "deeper" or "shallower", turn them upside down-that is, to translate, dilate and reflect parabolas.

I began to teach students in small groups about quadratic functions on a "need to know" basis, and the knowledge spread quickly around the class as students admired the more realistic look of the improved games. In two or three class sessions, the whole class had learned a topic that would normally take twice as long in Grade 10 math. What's more, learning made sense in the context of the fulfillment of a desire and a resulting aesthetic sense of satisfaction.

\section{Description of the Project}

In this new project on secondary mathematics teaching reform, an interdisciplinary team of educators, computer scientists, cognitive scientists and kinesiologists has come together to address the need for a radically different approach to secondary school mathematics that meets students where they live and in the ways that they learn. The current shorthand title for the project is Haptic Math, or Multimodal Math as a Design Tool. It involves a number of related initiatives with a basis in mathematical embodiment, arts-infused curriculum in mathematics, multisensory representations of mathematical relationships, and movement among various connected software/virtual worlds and physically-present worlds. The project puts mathematics at the heart of a 
variety of open-ended on- and off-line design projects, treating mathematical patterning as a basis for design and analysis in visual, auditory and tactile realms.

To my knowledge this is the first coordinated effort to create a body of sensory and design-based experiences for curricular topics in secondary school mathematics. A key innovation will be the use of haptic (tangible) interfaces along with audible/musical and 2D and 3D manipulable visual interfaces. An important principle in the software design will be the availability of translation of mathematical representations from one sensory mode to another and from one computer application to another.

Some "what-if" or hypothetical questions guiding the design process for this project include the following:

- What if the math in the secondary school curriculum were interwoven with sensory images - visual, tactile and auditory?

- What if students could move fluidly among algebraic and sensory representations of mathematical relationships; and if changing one representation (sensory or algebraic) changed all of them correspondingly?

- What if students had the chance to import mathematical objects from their math studies into other digital applications so that the math could be used with purpose-in the design of games, movies, web pages, desktop publishing?

- What if a CAD program allowed students to make actual physically present objects, of wood, metal, plastic from their mathematical designs?

Teaching mathematics with software that allowed for these kinds of interactions would blur a number of boundaries that have been significant in the history of mathematics and math education: disciplinary boundaries (math vs. applied skills, music, art for example); distinctions between "teacher as knower" and "student as listener" (already challenged by the use of computer networks in schools); and perhaps most significantly, mind/ body boundaries that have played a central role in shaping the public image of mathematics for millennia.

Distinctive features of this new project include the following:

- Learning software will be coordinated with the topics included in mandated provincial secondary school math curricula.

- Haptic software will be aimed at all students, not only those with visual difficulties.

- There will be built-in interactivity between various sensory and algebraic representations, so that learners can change any one representation and thus change all of them, highlighting their structural equivalence.

- Fluid movement between virtual, abstract and physically present representations will be facilitated by the use of CAD to make physical models. 
- The software will encourage the importation of mathematical virtual objects into other applications: music authoring, animation, web authoring, desktop publishing and games authoring software.

- We will explore other kinesthetic interfaces (for example, riding a programmed exercise bike up and down the graph of a function).

- We will explore miniature embedded haptic interfaces in physical objects away from the computer screen.

The following three key facets of the project are being developed:

1. A variety of sensory interfaces for mathematics students including haptic (i.e., tangible, force-related), kinesthetic, musical, auditory and visual computer interfaces.

2. Whole-body engagement with mathematical conceptualization, integrating gesture, movement and dance with the mathematics curriculum.

3. Using mathematical concepts from the secondary school curriculum as the basis for student-initiated design in a wide variety of creative realms. Many of these areas of design now have their basis in computer software, and this project aims to create a fluid movement among mathematical conceptualizations and representations (as a core for design) and the various software and fabrication applications used in design (Sterling 2005). The aim is a fluid two-way movement between mathematics and design (where changes in the mathematics affect the design and viceversa), engaging embodied, virtual and imaginary worlds.

\begin{tabular}{|l|l|}
\hline Facet of the Project & Specific Initiatives \\
\hline Variety of sensory interfaces & - The "Twiddler" haptic interface for graphing functions \\
& $\begin{array}{l}\text { - Riding an exercise bike over a graph } \\
\text { - Dance Dance revolution as an interface for learning number } \\
\text { - theory and functions }\end{array}$ \\
\hline Whole-body engagement & - Reading graphs with the body \\
- Rraph, gesture \& bodily images \\
- "Graph dance" \\
- Walking least common multiples \\
- Time \& function \\
- Math, body \& emotion
\end{tabular}




\section{Conclusions}

As rapid technological change transforms our culture in profound ways, young people particularly begin to adapt to the new "worlds" that seamlessly integrate virtual and physically-present environments. In this newly-emergent postmodern culture, boundaries of all kinds begin to dissolve, and what were once hard-andfast lines (between work and play, producer and consumer, arts and sciences, individuals and society, teachers and learners) become blurred.

As high school students (and their teachers) swiftly acculturate to the "Web 2.0" environment of social networking and widely available convergent creative software, collaboration, bricolage and a creative commons becomes our normal way of being. To block this "flow" at the door of the math classroom, out of conservatism or a paucity of imagination for new pedagogies, is to miss out on tremendous opportunities to afford young people with opportunities for engagement with mathematical ideas.

A by-product of our immersive and enriched virtual living spaces is a new appreciation for embodiment as an art form, and a new recognition of the importance of bodily, "hands on” kinesthetic experience in learning processes. In particular, it has been recognized that the abstract patterns of mathematics are rooted in our physical and perceptual experiences and continually draw nourishment and grounding from these experiences. The classical/modernist valuing of mental processes detached from physical being is a concept that has become obsolete.

The new Haptic Math, or Multimodal Math as a Design Tool collaborative project at UBC is addressing these profound cultural changes through the development of software, hardware, teaching methods and theory for secondary mathematics, supporting a variety of sensory inputs, whole-body engagement, and math-based student design. The multi-year project, which involves researchers from education, computer science, cognitive science and kinesiology, is in its initial phases but is already beginning to develop computer applications and teaching protocols for use in mathematics classrooms.

\section{References}

Berry, K. S. (2004). Bricolage is many a new thing understood. In Kincheloe, J. L. \& Berry, K. S. (Eds.), Rigour and complexity in educational research: Conceptualizing the bricolage (pp. 147-169). New York: Open University.

Borba, M. \& Scheffer, N. (2003). Sensors, body, technology and multiple representations. Proceedings of the $27^{\text {th }}$ Annual Meeting of the International Group for the Psychology of Mathematics Education (PME27), Hawaii, United States, 121-126.

Burton, L. (2001). Research mathematicians as learners-and what mathematics education can learn from them. British Educational Research Journal, 27(5), 589 - 599.

Davis, P. J. \& Hersh, R. (1981). The mathematical experience. Boston: Houghton Mifflin.

Gerofsky, S., Sinclair, N., \& Davis, B. (2002). Mathematics and the arts. In E. Simmt \& B. Davis (Eds.), Canadian Mathematics Education Study Group (CMESG) Conference Proceedings (pp. 33-42). Edmonton: University of Alberta.

Haraway, D. (1985). A manifesto for cyborgs. Socialist Review, 80.

Henderson, D. W. \& Taimina, D. (2007). Experiencing meanings in geometry. In Sinclair, N., Pimm, D., \& Higginson, W. (Eds.), Mathematics and the aesthetic: New approaches to an ancient affinity, (pp. 58-83). New York: Springer-Verlag.

Hewitt. D. (1996). Mathematical fluency: The nature of practice and the role of subordination. For 
the Learning of Mathematics, 16(2), 28-35.

Lakoff, G. \& Nuñez, R. E. (2000). Where mathematics comes from: How the embodied mind brings mathematics into being. New York: Basic Books.

Lappas, D. \& Spyrou, P. (2003). Embodied cognition and the origins of geometry: A model approach of embodied mathematics through geometric considerations. arXiv:math/0308003v1, $15 \mathrm{pp}$.

McLuhan, M. \& McLuhan, E. (1988). Laws of media: The new science. Toronto: University of Toronto Press.

Menzies, H. (2005). No time: Stress and the crisis of modern life. Vancouver, British Columbia: Douglas \& McIntyre.

National Council of Teachers of Mathematics (NCTM). (2000). Principles and Standards for School Mathematics. Reston, VA: NCTM.

Nemirovsky, R. (2003). Three conjectures concerning the relationship between body activity and understanding mathematics. Proceedings of the $27^{\text {th }}$ Annual Meeting of the International Group for the Psychology of Mathematics Education (PME27), Hawaii, United States, 105109.

Nuñez, R. E. (2000). Mathematical idea analysis: What embodied cognitive science can say about the human nature of mathematics. Proceedings of the $24^{\text {th }}$ Annual Meeting of the International Group for the Psychology of Mathematics Education (PME24), Hiroshima, Japan, 3-22.

Nuñez, R. E., Edwards, L., \& Matos, J.F. (1999). Embodied cognition as grounding for situatedness and context in mathematics education. Educational Studies in Mathematics, 39, 45-65.

Pimm, D. \& Jackiw, N. (2002). Mathematics, the written and the drawn. In E. Simmt \& B. Davis (Eds.), Canadian Mathematics Education Study Group (CMESG) Proceedings (pp. 71-79). Edmonton: University of Alberta.

Radford, L. (2003). Gestures, speech and the sprouting of signs: A semiotic-cultural approach to students' types of generalization. Mathematical Thinking and Learning, 5(1), 37-70.

Ramloll, R., Yu, W., Brewster, S., Riedel, B., Burton, M., \& Dimigen, G. (2000). Constructing sonified haptic line graphs for the blind student: first steps. Proceedings of $4^{\text {th }}$ International ACM conference on Assistive Technologies, Arlington, VA, 17-25.

Roth, P., Petrucci, L., \& Pun, T. (2000). "From dots to shapes”: An auditory haptic game platform for teaching geometry to blind pupils. Proceedings of the $7^{\text {th }}$ International Conference on Computers Helping People with Special Needs, pp. 603-610.

Schroeder, C. \& Gerofsky, S. (1998). Beyond the span of my limbs: Gesture, number and infinity. Journal of Curriculum Theorizing, 14(3), 39-48.

Sinclair, N., Pimm, D., \& Higginson, W. (Eds.) (2007). Mathematics and the aesthetic: New approaches to an ancient affinity. New York: Springer-Verlag.

Sinclair, N. (2005). Chorus, colour, and contrariness in school mathematics. THEN: Journal, 12.

Skemp, R. (1976). Relational understanding and instrumental understanding. Mathematics Teaching, 77, 20-26.

Sterling, B. (2005). Shaping things. Cambridge, MA: MIT Press.

Tall, D. O. \& Thomas, M. (1991). Encouraging versatile thinking in algebra using the computer. Educational Studies in Mathematics, 22(2), 125-147.

Tall, D. O. (2002). Differing modes of proof and belief in mathematics. Proceedings of the International Conference of Mathematics: Understanding Proving and Proving to Understand, National Taiwan Normal University, Taipei, Taiwan, 91-107.

Tall, D. O. (2003). Using technology to support an embodied approach to learning concepts in mathematics. In L.M. Carvalho \& L.C. Guimaraes (Eds.), Historia e Tecnologia no Ensino da Matematica (Vol. 1, 1-28). Rio de Janeiro, Brasil.

Tall, D. O. (2004). Building theories: The three worlds of mathematics. For the Learning of Mathematics, 24(1), 29-32.

Thurston, W. P. (1995). On proof and progress in mathematics. For the Learning of Mathematics, 15(1), 29-37.

Treviranus, J. (1999). Adding feeling, sound and equal access to distance education. Retrieved January 10, 2006, from www.utoronto.ca/atrc/rd/library/papers/TREVIR_J.html 
Van Scoy, F. L., Kawai, T., Darrah, M., \& Rash, C. (2001). Haptic display of mathematical functions for teaching mathematics to students with vision disabilities: Design and proof of concept. Lecture Notes in Computer Science, 2058, 31 - 38.

Van Scoy, F., McLaughlin, D. \& Fullmer, A. (2005). Auditory augmentation of haptic graphs: Developing a graphic tool for teaching precalculus skill to blind students. Proceedings of the $11^{\text {th }}$ Meeting of the International Conference on Auditory Display, 5.

Watson, A. \& Tall, D. O. (2002). Embodied action, effect and symbol in mathematical growth. Proceedings of the $26^{\text {th }}$ Annual Meeting of the International Group for the Psychology of Mathematics Education (PME26), Norwich, United Kingdom, 369-376.

Whitely, W. (1999). The decline and rise of geometry in $20^{\text {th }}$ Century North America. In M. John Grant (Ed.), Canadian Mathematics Education Study Group (CMESG) Proceedings (pp. 730). St. John's, Newfoundland: Memorial University of Newfoundland.

Whiteley, W. (2002). Teaching to see like a mathematician. Paper presented at the Visual Representations and Interpretations Conference (VRI 2002), Liverpool, UK.

Whiteley, W. (2004). Visualization in mathematics: Claims and questions towards a research program. Paper presented at the $10^{\text {th }}$ International Congress of Mathematical Education, Copenhagen, Denmark. 